\title{
Addressing Nutrition and Food Insecurity During Pandemic Threats
}

ISSN: 2640-9208

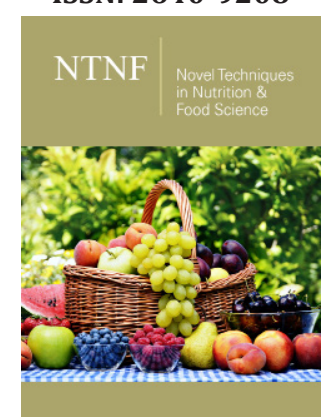

*Corresponding author: Tahir Turk, Adjunct Professor, Dow University of Health Sciences, Australia

Submission: 酶January 21, 2021

Published: 䀝February 17, 2021

Volume 5 - Issue 4

How to cite this article: Tahir Turk Addressing Nutrition and Food Insecurity During Pandemic Threats. Nov Tech Nutri Food Sci. 5(4). NTNF. 000619. 2021. DOI: 10.31031/NTNF.2021.05.000619

Copyright@ Tahir Turk. This article is distributed under the terms of the Creative Commons Attribution 4.0 International License, which permits unrestricted use and redistribution provided that the original author and source are credited.

\section{Tahir Turk*}

Adjunct Professor, Dow University of Health Sciences, Australia

\section{Commentary}

The Covid-19 pandemic has focussed resources in all countries globally, toward countering the pandemic threat. Despite the high infection and mortality rates in a number of high-income countries, social and economic disparities determine that the impact of Covid-19 will be most acute in low -and middle-income countries. These challenges are exacerbated in humanitarian situations in the poorest and most fragile economies where access to nutritious food, clean drinking water, and good quality health services are limited, and the resources and capacities of caregivers are already stretched. Conflict, internally displaced populations and environmental factors further impact on food insecurity. In these settings, malnutritionstunting, wasting and hidden hunger-was already a growing problem before Coronavirus appeared with the rechannelling of donor resources to tackle the pandemic potentially weakening responses to nutrition and other public health priorities.

Global estimates (2019) identify 47 million children under 5 are wasted, of which 14.3 million are severely wasted, with 144 million being stunted. Around $45 \%$ of deaths among children under 5 years of age are linked to undernutrition, which mostly occurs in LMICs. Child stunting and malnutrition is also associated with lower dietary diversity, with food insecurity and poor household food choices contributing to the lack in meeting children's nutrient requirements. Additionally, malnutrition is seen to predominantly occur in regional hotspots where there is limited access to high protein foods, poor vegetation cover, and a proxy of rainfall or drought. Malnutrition is exacerbated with pandemic threats like HIV/AIDS, drug resistant TB and airborne viruses like Covid-19, which further restricts opportunities for income generation, which puts food into the mouths of the world's most vulnerable populations. UNICEF reports that in nearly every part of the world, families face economic, political, market, social or cultural barriers to providing nutritious, safe, affordable and sustainable diets to young children with socioeconomic determinants playing a significant role in food insecurity and diversity of food choices, leading to malnutrition.

These risk factors indicate that rather than weakening responses toward nutrition interventions to address food insecurity, the pandemic should instead increase our vigilance to better understand the social and behavioral determinants supporting interventions to build greater resilience, during these times of high need. A number of agencies including UNICEF, FAO, GAIN, World Bank and regional development partners like the Southern African Development Community (SADC) have continued to consult with stakeholders and develop strategic approaches to address food insecurity and malnutrition in many parts of the world. During this time, innovative approaches have been developed, given lockdowns and travel restrictions caused by the pandemic. The application of virtual support and capacity building of local counterparts to implement field research and data collection has generated important program intelligence. Needs assessments, implemented in a number of settings during the height of the Covid-19 pandemic, have demonstrated that priority nutrition program strategic planning can continue, while the health sector deals with other global health priorities.

The adaptation to virtual support provided a number of learnings including the potential for technical capacity building, data collection and analysis, albeit over an extended 
timeframe given the need for partner communication over Zoom and other online applications. As a result, a number of innovative approaches have been identified along with opportunities for national and regional efficiencies in adapting existing, best practice creative approaches, rather than 'reinventing the wheel'. Strategy development continues with opportunities to better utilize social and behavior change communication (SBCC) and piloting and evaluation of SBCC nutrition interventions for their efficacy, with the aim to scale-up to achieve population level impact. These efforts must continue as the pandemic rages around the world, and well after the vaccines have been distributed. It is incumbent on donors, regional partners and governments of fragile member states to maintain advocacy to address priority health programs, including nutrition, if broader health gains are ultimately to be achieved. 